\title{
Cambios en los significados de la masculinidad en hombres del sur de Chile
}

\section{Changes in the meaning of masculinity in men of southern Chile}

\author{
Ana María Poo ${ }^{1}$ y Beatriz Vizcarra ${ }^{2}$ \\ 'Psicóloga. Magister en Pedagogía y Gestión Universitaria. Académica del Departamento de \\ Psicología, Universidad de La Frontera. E-mail: ana.poo@ufrontera.cl
}

${ }^{2}$ Psicóloga. Doctora en Educación, Académica del Departamento de Psicología, Universidad de La Frontera. E-mail: maría.vizcarra@ufrontera.cl

\begin{abstract}
Proyecto financiado por la Dirección de Investigación de la Universidad de La Frontera, Temuco, Chile.

Las autoras agradecen a la Doctora Pamela Jiménez, Académica del Departamento de Psicología de la Universidad de La Frontera, por la revisión del manuscrito.

Departamento de Psicología, Facultad de Educación, Ciencias Sociales y Humanidades, Universidad de La Frontera.

Temuco, Chile.
\end{abstract}

\section{Resumen}

En América Latina, los estudios sobre masculinidades e intervención con hombres, desarrollados en las últimas décadas, han buscado conocer las subjetividades y prácticas, así como los modelos y mandatos de la masculinidad, visibilizando la participación de los hombres en algunas desigualdades de género (Aguayo y Nascimento, 2016; Olavarría, 2017). Si bien estos estudios muestran una evolución en el modelo tradicional de masculinidad, en América Latina, y en especial en Chile, existen brechas de conocimiento acerca de las diferencias en las representaciones de la masculinidad en distintas generaciones y contextos sociales (Aguayo y Nascimento, 2016). El objetivo del presente estudio fue describir los significados de la masculinidad en hombres del sur de Chile, comparando generaciones y niveles socioeconómicos. Se utilizó un diseño de casos múltiples; la muestra estuvo constituida por jóvenes
(18 a 24 años) y adultos medios (45-69 años) distribuidos en tres niveles socioeconómicos. Se desarrollaron seis grupos de discusión. Para el análisis de la información se llevó a cabo un primer nivel de codificación abierta, estableciendo unidades de significado. Estas unidades fueron transformadas por medio de un proceso inductivo en categorías, las cuales fueron comparadas para identificar similitudes, diferencias y posibles vínculos entre ellas. Se encontraron coincidencias y diferencias en los significados asociados a la masculinidad, en función de la edad y del nivel socioeconómico. Las coincidencias en función de la edad se dieron principalmente en las categorías Identidad y Trabajo, y las mayores diferencias en el núcleo Sexualidad, mientras que en función del nivel socioeconómico la mayor diferencia estuvo en la categoría Trabajo.

Palabras clave: masculinidad; hombres; significados; cambios; chilenos. 


\section{Abstract}

In Latin America, studies on masculinities and intervention with men developed in recent decades have sought to know the subjectivities and practices, as well as the models and mandates of masculinity, making visible the participation of men in some gender inequalities (Aguayo y Nascimento, 2016; Olavarría, 2017). Although these studies show an evolution in the traditional model of masculinity, in Latin America and in Chile, there are knowledge gaps about differences in the representations of masculinity in different generations and social contexts (Aguayo y Nascimento, 2016). The purpose of this study was describing the meanings of masculinity in men from southern Chile, comparing generations and socioeconomic levels. A multiple case design was used, sample consisted of 44 Chilean men, young men between 18 and 25 years, and middle adults between 45-69 years, distributed in 3 socioeconomic: levels, high, medium and low. Six discussion groups were developed around a set of guiding questions, built by the authors, in relation to the mandates of masculinity: identity referred to the characteristics that the participants consider essential to masculinity, sexuality, paid work and paternity. Information obtained was transcribed the first level of open coding was then carried out, which consisted in selecting units of meaning which emerged from the answers to the guiding questions. These units, consisting of paragraphs, were transformed into categories by an inductive process, each category being given a code representing its meaning. The second level of coding -at a greater level of abstraction- consisted in comparing the categories, identifying similarities, differences and possible links between them. Results, in the first nucleus, Identity, arise as main categories: Concept in Transition, Difficulty of Being Men, Action-Oriented, Restriction in the Expression of Emotions, Integrity, Masculinity given by the Biological Condition. In the second nucleus Work: Provide Identity Trait of Masculinity, Facilitator of Independence, Source of Self-Realization and Dignification, Obstacle for the Satisfaction of Other Needs, Labor Discrimination towards Women. In the third nucleus, Sexuality, categories differentiated by age, in young people emerge Acceptance of Homosexuality, Stereotypes in Sexual Behavior and Increase in Sexual Demands. In adults Divergences against the Mandate of Heterosexuality and in both groups Sexual Behavior in Transition. On the other hand, coincidences and differences were found in the meanings associated with masculinity, depending on age and socioeconomic status. The coincidences in the Identity nucleus, realize that men, regardless of their age or socioeconomic condition, experience a situation of accelerated change in their roles, which affects them to a greater or lesser extent, which is experienced by the majority of young people as a possibility of transcending stereotypes, allowing them greater degrees of freedom. Regarding the core Work, however, the broad coincidence in all groups in relation to the mandate of being a provider, certain nuances are appreciated, for adults it is an internal mandate, a duty of masculinity, while for young people it seems respond to an external social demand, which accounts for a masculinity in transition. The differences, by age range were the nucleus Sexuality, where young people have more openness to address their own sexuality and more inclusive behaviors against homosexuality. Differences relating socioeconomic level occurred in the nucleus Work between young people and adults. If in the young people of high and medium socioeconomic level, work implies the possibility of becoming independent, for youngsters of low socioeconomic level, it also means taking care of their parents, responding to a duty of reciprocity. In adults of low socioeconomic level, unlike those in the medium and high level, the centrality and physical requirement of work displaces others needs as health care and sexual life.

Keywords: masculinity; men; meanings; changes; Chilean men. 


\section{Introducción}

Las familias, los varones y las masculinidades están en plena transformación. De acuerdo con estudios desarrollados en EE.UU., Canadá y diversos países europeos, actualmente se asiste a una revolución del género que se expresa en dos procesos de transformaciones sucesivas: la primera con la salida de las mujeres del espacio doméstico e ingreso al mundo laboral y la esfera pública, y la segunda con el involucramiento de los hombres en las tareas domésticas y el cuidado de los niños (Aguayo, Correa y Cristi, 2011; Goldscheider, Bernhardt y Lappegard, 2014). Estas transformaciones se expresan igualmente en el creciente interés, en los últimos 40 años en el mundo desarrollado, en los estudios de las masculinidades e intervenciones con hombres (Connell, 2011; Kimmel y Messner, 2004; Olavarría, 2003).

Las conceptualizaciones de la masculinidad son diversas y varían de acuerdo con los contextos sociohistóricos. La masculinidad se ha definido como un conjunto de valores, atributos, conductas, roles e identidades de los hombres que se suponen esenciales en el varón en una cultura determinada (De Keijzer, 2010; Goldscheider et al., 2014). Palermo (2016), por su parte, describe la masculinidad como el ordenamiento social e histórico a través del cual los hombres se comprometen en una posición de género y clase, generando efectos concretos en las prácticas, experiencias y representaciones acerca de lo masculino y lo femenino. La coexistencia de múltiples posibilidades de ser, actuar e identificarse como un hombre refleja la evolución de las masculinidades según las culturas y los períodos históricos, pero también su carácter variable durante la vida de los individuos (Connell y Messerschmidt, 2005; Kimmel y Messner, 2004).

Históricamente han existido distintos paradigmas o marcos interpretativos para abordar el estudio de la masculinidad (Kilmartin, 2007; Addis y Cohane, 2005). Una primera corriente teórica se centra en los procesos de socialización de género de los hombres y el impacto de los roles sobre la salud física y mental (De Keijzer, 2001; O'Neil, 2008). Un segundo enfoque, que comprende gran parte de la producción científica en este campo, se inscribe en una perspectiva crítica de las masculinidades y se orienta a la comprensión de las dinámicas de poder entre hombres y mujeres, y entre los mismos hombres. Desde este enfoque surge el concepto de "masculinidad hegemónica", la cual sería el modelo que hay que seguir para ser considerado un hombre, generalmente asociado a varones heterosexuales que monopolizan el poder, el prestigio y la autoridad legítima. Este tipo de masculinidad estaría en una condición de superioridad respecto de las mujeres, así como de las masculinidades subordinadas o marginadas de la posición de poder, frecuentemente asociadas a los homosexuales o grupos étnicos minoritarios (Connell, 2005; Connell y Messerschmidt, 2005; Kaufman, 1997; Kimmel, 1997).

Un tercer paradigma propone aprehender el género y las masculinidades como una performance de sí mismo en el seno de contextos altamente normativos, destacando la posibilidad, si no la necesidad, de deconstruir o reinventar el género (Butler, 2006; Petersen, 2003). Los estudios relacionados con los paradigmas más recientes sitúan a los hombres como sujetos dentro de un sistema sexo-género, de ideologías, identidades y relaciones androcéntricas y heterosexistas.

Peretz (2016) plantea que la relevancia de los estudios de masculinidad bajo estos paradigmas radica en que una mejor comprensión de ella contribuye a develar la condición de superioridad de los hombres y las masculinidades, a desnaturalizar dicha superioridad, favoreciendo la disminución de la violencia de género e incrementando la justicia de género. Por otra parte, y en concordancia con Barker y Greene (2011), los estudios acerca de si los varones no deberían tener solo como objetivo reparar las desigualdades que enfrentan las 
mujeres también deberían ser estudiados para describir, desde sus vivencias, sus propias necesidades y vulnerabilidades relacionadas con el género, para ser incluidas en las políticas públicas. En ello radica la relevancia del presente estudio.

En América Latina, los estudios sobre masculinidades e intervención con hombres se han desarrollado básicamente en las tres últimas décadas. En esta producción se ha buscado conocer las subjetividades y prácticas de masculinidades diversas, sus modelos y mandatos, y se han desarrollado conceptualizaciones y categorías para abordar e intervenir en dichas inequidades, por lo que se ha visibilizado la participación de los hombres en algunas desigualdades de género. De esta manera, se ha ido transitando de las evidencias a la incidencia en políticas y normativas de igualdad de género. Al mismo tiempo, se ha reflexionado sobre cómo convocar e involucrar a los hombres en actitudes y comportamientos más equitativos, cuestionando el modelo hegemónico de masculinidad que sirve de base para discriminar y subordinar a la mujer (Aguayo y Nascimento, 2016; Olavarría, 2017). A pesar de la vigencia de este modelo en algunos sectores sociales de América Latina, el mundo masculino actual es más heterogéneo y se mueve hacia la diversidad. Este fenómeno se observa particularmente en ambientes urbanos y entre varones jóvenes o de mediana edad, con un nivel educativo medio-alto o alto que han tenido con frecuencia experiencias vitales con algún referente de igualdad, ya sea discursivo y/o simbólico (Aravena y Baeza, 2010; Fuller, 2005; Matamala y Rodríguez, 2010).

Si bien estos estudios muestran una evolución en el modelo tradicional de la masculinidad, tanto en Chile como en el resto de América Latina, aún existen brechas de conocimiento acerca de las diferencias en sus representaciones en las distintas generaciones y contextos sociales (Aguayo y Nascimento, 2016). Considerando que las identidades son un constructo relativo al contexto sociohistó- rico (Iñiguez, 2001), se espera que los hombres jóvenes chilenos, que nacieron y crecieron en democracia y en medio de la globalización, construyan discursos más equitativos, comparados con los adultos que vivieron gran parte de su adultez en una sociedad caracterizada por la represión y la censura bajo la dictadura militar. Por otra parte, como señala Connell (2003), todas las esferas de la identidad que construyen los varones, incluso la relación de pareja y la sexualidad, están influenciadas por las circunstancias laborales, los lugares de trabajo, las condiciones económicas y las estructuras de las organizaciones, por lo que se espera que varones provenientes de sectores populares se adscriban a modelos más tradicionales de masculinidad en comparación con los provenientes de sectores medios y altos. Estas brechas no han sido suficientemente estudiadas en Chile, y la producción todavía es muy escasa en cuanto a la relación entre masculinidades. Sin construir este conocimiento, difícilmente las políticas de igualdad de género, que intervienen con hombres, podrán ser adecuadamente focalizadas y tener pertinencia cultural.

Basado en los antecedentes expuestos, la presente investigación se propuso como objetivo general explorar las similitudes y las diferencias en el significado de la masculinidad construido por hombres adultos y hombres jóvenes, de distinto nivel socioeconómico de la ciudad de Temuco (Aguayo y Nascimento, 2016).

A partir de ello, se plantearon los siguientes objetivos específicos:

1) Describir el sentido de la masculinidad en función de la identidad, el trabajo y la sexualidad construido por hombres jóvenes y adultos.

2) Comparar el sentido de la masculinidad en función de la identidad, el trabajo y la sexualidad construido por hombres adultos de distinto nivel socioeconómico.

3) Comparar el sentido de la masculinidad en función de la identidad, el trabajo y la sexualidad construido por hombres jóvenes de distinto nivel socioeconómico. 


\section{Método}

\section{Diseño}

Se utilizó un diseño cualitativo de casos por criterio considerando el grupo etario con dos valores (jóvenes y adultos) y el nivel socioeconómico con tres valores: nivel socioeconómico bajo (NSEB), nivel socioeconómico medio (NSEM) y nivel socioeconómico alto (NSEA) cuyo cruce genera una matriz de seis observaciones.

\section{Participantes}

La población de estudio fueron hombres chilenos, jóvenes entre 18 y 24 años y adultos entre 45 y 69 ; no se incluyeron varones entre 25 y 49 años con el propósito de relevar las diferencias generacionales. Para determinar la variable socioeconómica, se utilizó como proxy el nivel educacional alcanzado en los adultos y en los jóvenes el nivel educativo alcanzado por ambos padres: NSEB con 8 o menos años de escolaridad, NSEM, con 9 a 15 años de educación formal y NSEA con más de 17 años de educación formal.

Utilizando la técnica de muestreo intencionado, se convocó la conformación de seis grupos de conversación, para responder así a la matriz definida en el diseño, y quedó la muestra final constituida por esos grupos, en los que participaron 44 hombres entre 18 y 69 años.

\section{Técnicas}

Como técnica de recolección de datos se utilizó el grupo de discusión, puesto que permite acceder a los significados que los participantes construyen grupalmente acerca de determinados tópicos a partir de sus experiencias, creencias y emociones. La discusión grupal se organizó en base a una pauta de preguntas guía, confeccionada por las autoras, en función de tres núcleos asociados a la masculinidad: (1) la identidad, comprendida como la percepción individual que una persona tiene sobre sí misma; la conciencia del existir, adquirida a lo largo de la vida, que moldea la conducta y la personalidad (Vera y Valenzuela, 2012); (2) la sexualidad, comprendida como el resultado de la interacción de factores biológicos, psicológicos, socioeconómicos, culturales, éticos y religiosos o espirituales que se expresa en todo lo que somos, sentimos, pensamos y hacemos (OMS, 2006), y (3) el trabajo, considerado como una actividad relevante de orden social que permite estar o considerarse parte de un sistema organizado y ser llamados "ciudadanos" (Alonso, 2005, citado por Romero, 2017). Esta guía sirvió como herramienta de apoyo y de recordatorio para la discusión de modo de asegurar que se cubrieran todos los temas (Taylor y Bogdan, 2004). Las preguntas fueron presentadas siguiendo una secuencia organizativa de las más generales a las más específicas.

\section{Procedimiento}

Para acceder a la muestra, se contactó a distintas instituciones educativas y sociales de la ciudad de Temuco en las cuales estudiaban o trabajaban hombres que se ajustaban a los perfiles requeridos por el estudio. Se realizó una amplia convocatoria considerando los criterios de inclusión para constituir los distintos grupos de estudio.

Los grupos se realizaron en forma secuencial en el transcurso de 6 meses. Estos fueron conducidos por una investigadora $\mathrm{y}$ un ayudante de investigación varón, $\mathrm{y}$ tuvieron una duración aproximada de 1 hora 45 minutos. En cuanto a los participantes, antes de iniciar la discusión, se les entregó un consentimiento informado en el que se les explicó que su participación seria anónima y voluntaria, que la discusión sería registrada a través de grabaciones de audio y notas de campo, y que podrían retirarse de la investigación en el momento que desearan y acceder 
a los resultados si lo solicitaban. Asimismo, se enfatizó el resguardo de los datos por parte de las investigadoras y que estos se utilizarían solo con fines de investigación.

El estudio se enmarca en el Proyecto Diufro D14-0002, el cual fue evaluado por el Comité de Ética de la Universidad de La Frontera.

\section{Plan de análisis}

La información obtenida fue transcripta y los participantes fueron identificados con un número para resguardar la confidencialidad. Luego se complementó con las notas de campo de las investigadoras y se revisó el documento para tener una visión general de los datos. Posteriormente, se llevó a cabo un primer nivel de codificación abierta que consistió en seleccionar unidades de significado que emergieron de las respuestas a la pauta de preguntas. Estas unidades que consistían en párrafos fueron transformadas por medio de un proceso inductivo en categorías, a las que se le asignó un código que representa su significado. El segundo nivel de codificación de mayor abstracción consistió en comparar las categorías, identificando similitudes, diferencias y posibles vínculos entre ellas. En este proceso, las categorías fueron reduciéndose hasta llegar a los elementos centrales del análisis (Hernández, Fernández y Baptista, 2010).

Dentro de los criterios de rigor científico, durante el proceso de recolección de información, se validaron los datos por medio del uso de una pauta de preguntas para guiar la discusión grupal, registro de audio y notas de campo de las investigadoras y los ayudantes de investigación, y se utilizaron las transcripciones textuales para respaldar los significados e interpretación de los resultados. Además, se realizó un contrachequeo con los participantes. La validación de los resultados se aseguró mediante triangulación por investigadora en el proceso de codificación y análisis de datos, y por medio de comprobabilidad mediante revisión externa.

\section{Resultados}

En la Tabla 1 se presentan las características de los grupos que constituyen la muestra.

\section{Tabla 1}

Caracterización de la muestra.

\begin{tabular}{|c|c|c|c|c|c|c|}
\hline Grupos & $\begin{array}{c}\text { Número de } \\
\text { participantes }\end{array}$ & $\begin{array}{c}\text { Rango } \\
\text { de edad }\end{array}$ & Institución & $\begin{array}{c}\text { Nivel de } \\
\text { estudio }\end{array}$ & $\begin{array}{c}\text { Nivel } \\
\text { socioeconómico }\end{array}$ & Ocupación \\
\hline 1 & 9 & $\begin{array}{c}18-22 \\
\text { años }\end{array}$ & $\begin{array}{c}\text { Escuela para } \\
\text { Adultos }\end{array}$ & $\begin{array}{c}\text { Enseñanza } \\
\text { media } \\
\text { incompleta }\end{array}$ & Bajo & Estudiantes \\
\hline 2 & 9 & $\begin{array}{c}19-24 \\
\text { años }\end{array}$ & Universidad & $\begin{array}{c}\text { Enseñanza } \\
\text { superior } \\
\text { incompleta }\end{array}$ & Medio & Estudiantes \\
\hline 3 & 4 & $\begin{array}{c}19-24 \\
\text { años }\end{array}$ & $\begin{array}{c}\text { Instituto } \\
\text { Nacional de } \\
\text { la juventud }\end{array}$ & $\begin{array}{c}\text { Enseñanza } \\
\text { media } \\
\text { completa }\end{array}$ & Alto & $\begin{array}{c}\text { Estudiantes, } \\
\text { trabajadores } \\
\text { part-time }\end{array}$ \\
\hline 4 & 8 & $\begin{array}{c}45-55 \\
\text { años }\end{array}$ & $\begin{array}{c}\text { Empresa } \\
\text { constructora }\end{array}$ & $\begin{array}{c}\text { Enseñanza } \\
\text { media } \\
\text { incompleta }\end{array}$ & Bajo & Obreros \\
\hline 5 & 6 & $\begin{array}{c}45-60 \\
\text { años }\end{array}$ & Universidad & $\begin{array}{c}\text { Enseñanza } \\
\text { media } \\
\text { completa }\end{array}$ & Medio & $\begin{array}{c}\text { Auxiliares } \\
\text { administrativos }\end{array}$ \\
\hline
\end{tabular}




\begin{tabular}{|c|c|c|l|c|c|c|}
\hline Grupos & $\begin{array}{c}\text { Número de } \\
\text { participantes }\end{array}$ & $\begin{array}{c}\text { Rango } \\
\text { de edad }\end{array}$ & Institución & $\begin{array}{c}\text { Nivel de } \\
\text { estudio }\end{array}$ & $\begin{array}{c}\text { Nivel } \\
\text { socioeconómico }\end{array}$ & Ocupación \\
\hline 6 & 8 & $\begin{array}{c}45-69 \\
\text { años }\end{array}$ & Universidad & $\begin{array}{c}\text { Enseñanza } \\
\text { superior } \\
\text { completa }\end{array}$ & Alta & $\begin{array}{c}\text { Académicos } \\
\text { universitarios }\end{array}$ \\
\hline
\end{tabular}

Los resultados se presentan en función de tres de los cuatro núcleos centrales abordados en la presente investigación y de acuerdo con el significado otorgado por los participantes: Identidad, Trabajo, Sexualidad. Dada su extensión, los resultados relacionados con el núcleo Paternidad fueron descriptos en un artículo separado.

En el primer núcleo, Identidad, surgen como principales categorías: Concepto en transición, Dificultad de ser hombres, Orientados a la acción, Restricción en la expresión de emociones, Integridad, Masculinidad dada por la condición biológica. En el segundo núcleo, Trabajo, surgen como principales categorías: Proveer rasgo identitario de la masculinidad, Facilitador de la independencia, Fuente de autorrealización y dignificación, Obstáculo para la satisfacción de otras necesidades, Discriminación laboral hacia las mujeres.

En el tercer núcleo, Sexualidad, aparecen las categorías diferenciadas generacionalmente. En los jóvenes, Aumento de exigencias en el plano sexual, Aceptación de la homosexualidad, Estereotipos en el comportamiento sexual. En los adultos emerge la categoría Divergencias frente al mandato de la heterosexualidad y en ambos grupos etarios, Comportamientos sexuales en transición. A continuación, se describe cada núcleo con sus categorías.

El núcleo Identidad se refiere a las características que los participantes consideran esenciales o definitorias de lo masculino. En primer lugar, aparece la categoría Concepto en transición. Los varones de todos los grupos coinciden en que es difícil consensuar acerca de los elementos constitutivos de lo masculino, dada la magnitud de los cambios en los roles de género. Ello se expresa en las siguientes citas: "Hombre, hoy día, significa estar en una constante búsqueda de adaptación y flexibilidad, de poder asumir roles sin recibir el estereotipo de que eres marica porque estás haciendo lo que es de mujer..." (J-NSEA); "Hoy en día, yo creo que es difícil decir qué es un hombre porque tiene que ver con un cambio cultural de la construcción de la masculinidad o del género..., porque hace 20 años atrás hacías un grupo focal y era fácil decir 'yo soy hombre porque me gusta la cerveza fría', por ejemplo, o 'a mí me gusta ser hombre porque yo mando en la casa"" (J-NSEM); "La definición de hombre es una definición social, lo que significa ser un ser humano, que siempre existen designaciones desde fuera y no existe una identificación interior" (A-NSEA).

Una segunda categoría expresada por adultos de NSEA se refiere a la Dificultad de ser hombres, es decir, la masculinidad, a diferencia de la femineidad, es una condición por construir y está continuamente sometida a prueba: "Hombre es algo que se conquista, mucho más que el ser mujer" (A-NSEA); "No es fácil ser hombre; todos nosotros, desde cabros chicos, pasamos por duras pruebas en esto de la hombría, la masculinidad" (A-NSEA); "La condición masculina es tremendamente difícil de llevar, puesto que se está siempre a prueba" (A-NSEA); “...y lo peor es la exigencia social, el nivel de responsabilidad atribuida por el hecho de ser hombre y el nivel de exigencia de demostrar permanentemente que eres capaz y que soy hombre en todos los planos, yo siento que ha sido agotador, sigo en eso y ya asumí y dije 'soy hombre, tengo que asumirlo', claro, viene con el contrato, es genético" (A-NSEA)

En la tercera categoría, Orientados a la acción, se aprecia alto consenso en todos los grupos; esta se refiere a que lo masculino se define esencialmente a partir del hacer, los hombres se caracterizarían por la capacidad de liderazgo, logro de objetivos y resolución 
de problemas, y tendrían mayor tendencia a conducir, dirigir, conquistar, producir. El espacio natural de la expresión de estas características es el trabajo: "Dirigir, mandar, despedir, castigar, son roles supuestamente masculinos..." (A-NESA); “...presidenta, rectora, autoridad mujer que es masculina porque, en realidad, se vuelca al espacio público en busca de demostrar que tienen competencias" (A-NSEA); “...el hombre en sí es el macho alfa, en cierta forma, en los temas de cómo enfrentar ciertas situaciones" (A-NSEB); “... en cuanto al estilo de pensamiento y la forma de abordar, el hombre se centraba más en la meta, en el fin; en cambio la mujer cuando le presentaban el fin, el objetivo, se centraba más en el medio" (J-NSEM); “...el trabajo es una de las conquistas más importantes para demostrar ser hombre, demostrar que es competente y capaz" (A-NSEA).

La cuarta categoría Restricción en la expresión de emociones, se refiere a la dificultad para expresar emociones tanto placenteras como displacenteras. En esta categoría se advierten diferencias, ya que los adultos fueron más restrictivos que los jóvenes: “...para mí tiene que ver con que al hombre, hoy en día se le acepta más tener miedo, el hecho de poder sentir miedo" (J-NSEM); “...lo definiría como un sujeto que tiene que ser fuerte sí o sí, fuerte en el sentido de proteger a una mujer, proteger a una familia, pero también ahora con mayor afectividad hacia demostrar el lado emocional, pero no perdiendo la fortaleza que debe de tener para enfrentar algunas situaciones problemáticas" (A-NSEM); "El hombre, por eso, tiene que ser bien machito y aguantarse no más y confiar en el de arriba" (A-NSEB); "Nacemos bajo un estándar normativo, que nos exige tener un tipo de vinculación con nuestras emocionalidades, nuestras expresiones, en el cual el hombre tiene que mantener un cierto nivel de sobriedad, yo diría de un estándar de masculinidad, es decir, de no excederse en la expresión de su propio sentimiento, de su afectividad; entonces yo siempre he sentido que estoy cautivo dentro de un nicho formal, estructu- rado, cultural, que no me deja expresarme afectivamente como yo quisiera..." (A-NSEA); “... el hombre, aunque sea muy hombre, llora igual que un niño, porque uno trata de hacerlo, decir que no, pero a escondidas llora..."(A-NSEB)

La quinta categoría, Integridad, surge principalmente en los grupos de adultos, definiendo la masculinidad a partir de ciertos comportamientos como la responsabilidad, el respeto por los otros y la consistencia en el actuar: "Mi hombría yo la manifiesto respetando al otro y, obviamente, a la mujer, de esa forma me enseñó mi padre" (A-NESM); "Tener relaciones solamente con una mujer no significa que uno sea un hombre completo, aparte de eso hay que tener valores con ella, valores, la fidelidad y todo eso, eso es un hombre completo" (A-NSEB); “... ser hombre es cumplir la palabra" (J-NSEB); "...hay hombres que le pegan a la mujer, para mí eso es ser poco hombre" (J-NSEB).

Por último, aparece la categoría Masculinidad dada por la condición biológica, la cual alude a que la masculinidad finalmente se sustenta en la anatomía y fisiología masculina: “...lo que define ser hombre para mí en limpio y en puro, es una cuestión clínica biológica" (A-NSEA); “...tener el cromosoma Y..." (J-NSEA); “... efectivamente creo que hay una diferenciación biológica además de la diferenciación social” (A-NSEA); “...un hombre, que no puede ser madre” (A-NSEB); “...yo creo que es lo evidente, desde lo biológico, se diferencia por el aparato reproductivo, ciertas hormonas" (J-NSEM).

El núcleo Trabajo se define como la realización de funciones en un contexto formal por las cuales se recibe remuneración. La primera categoría que surge, Proveedor rasgo identitario de la masculinidad, es transversal en todos los grupos etarios y socioeconómicos. Se refiere a que el trabajo es el eje de la vida, implica la exigencia de ser proveedor principal, y no cumplir con este mandato es objeto de sanción social: "Es mal visto que la mujer salga a trabajar y el hombre se quede en la casa, no haciendo nada" (J-NSEM); "Es mal visto que te invite ella, puede ser a medias porque somos 
estudiantes, pero es mal visto que te invite" (J-NSEM); “ “...aun cuando las mujeres se han incorporado al trabajo, pero el hombre es como él, la persona que debe sustentar el hogar" (A-NSEM); “...casos en que la mujer tiene un buen trabajo, buen sueldo y el hombre se puede quedar en la casa a cargo de los niños, si lo hace es macabeo, mantenido, cafiche" (J-NSEA); "...no se siente hombre uno cuando no tiene trabajo, estar uno ahí, pucha, siendo mantenido por la pareja en algunos casos o simplemente el no tener pega es como que te cortan las alas, no se sientes hombre" (A-NSEB); “...el trabajo es la responsabilidad que toma uno cuando se junta con una mujer a vivir y formar una familia, porque uno sabe que formando una familia tiene que ser responsable en cuanto a los alimentos y cosas que tiene que llevar como hombre uno a la casa" (A-NSEB)

Una segunda categoría que aparece en jóvenes y adultos es Facilitador de la independencia, entendida como necesidad de los varones de hacerse cargo de sí mismos y eventualmente de otros: "...uno debe tener su independencia, su autonomía, su plata" (J-NSEM). "Conquista de la autonomía, de la libertad que le produce tener ingresos" (A-NSEA); “...por último si está viviendo hasta los 30 años con los padres, que no le compren hasta el desodorante, que se pueda mantener, aunque siga bajo el amparo de los padres" (J-NSEM); "Yo pienso que una obligación también es como devolverles la mano a los padres de todo lo que ellos han hecho cuando uno ya sea más grande y tenga plata, no sé, comprarle, darle regalos, lo que necesiten. Darles la mejor comodidad para que estén bien ellos" (J-NSEB).

La tercera categoría que surge en los adultos de NSEB es Obstáculo para la satisfacción de otras necesidades, y se refiere a que el trabajo, principalmente físico, demanda un alto nivel de energías que termina afectando el ejercicio de la sexualidad: “...lo que pasa que uno se mete mucho en la pega laboral y como que deja la sexualidad a un lado, como que no le toma mucho interés ya uno porque lo que interesa es trabajar no más” (A-NSEB); “...el trabajo, a algunas personas le pasa eso, le quitan mucho otras cosas, por ejemplo, el estar con la familia, lo mismo que llegan muy cansados a la casa y dejan de lado la parte sexual ...igual se afecta un poco a la pareja" (A-NSEB); "Entonces uno llega no más, una bañada, come y a descansar no más, pensando y ahí uno no se siente hombre porque queda pensando, chuta no estoy cumpliendo con mi señora" (A-NSEB).

La cuarta categoría, Fuente de autorrealización y dignificación, se refiere a que el trabajo satisface la necesidad de trascendencia y la valoración social. Esta categoría tiene más peso en el NSEM y NSEB: “...alcanzar autorrealización a través del trabajo" (J-NSEM); "el trabajo dignifica y se siente seguro" (J-NSEM); "...hay un dicho que dice que el trabajo dignifica a la persona, al hombre en este caso, teniendo trabajo uno puede hacer varias cosas" (A-NSEB).

La quinta categoría, Discriminación laboral hacia las mujeres, surge tanto en adultos como en jóvenes y se refiere a la percepción de desigualdad en las condiciones laborales de las mujeres en relación con los hombres: "Hay una discriminación clara, por ejemplo el hombre al hacer una labor que hace una mujer, va a ser mejor remunerado" (J-NSEM); "Ser hombre es existir con muchas más posibilidades, con las puertas mucho más abiertas, con muchos menos problemas que las mujeres... en los trabajos donde pueden haber los dos sexos, siempre hay hombres que ocupan los cargos más importantes" (A-NSEA); "Los grandes cargos, o jefes de una empresa de construcción, señalan que las mujeres no favorecen el trabajo...son más cacho o mucho más problema" (J-NSEM).

El tercer núcleo, Sexualidad, se refiere a las expectativas sociales respecto del comportamiento sexual masculino. Las categorías emergen diferenciadas por grupo etario $\mathrm{y}$ nivel socioeconómico. La primera categoría, Aumento de exigencias en el plano sexual, surge en los jóvenes y se relaciona con la percepción de empoderamiento de las mujeres, que trae como consecuencia mayor exigencia 
en el rendimiento sexual de los varones: “... por problemas de que la mujer considera que el hombre no le sirve para la cama, no le satisface sexualmente y eso anteriormente no eran problemas, para un hombre no eran problemas, porque era así nomás. Y hoy en día la mujer se da esa capacidad de ir, probar, escoger" (J-NSEM); "Creo que es un tema mucho más complicado para el hombre que para la mujer, o sea, que es el hombre el que no puede equivocarse, por ejemplo, el tema de la eyaculación precoz, la disfunción eréctil y eso involucra mucha presión para el hombre de satisfacer sexualmente a la mujer... y, en el fondo, la mujer no tiene la presión que tiene el hombre en el tema sexual"(J-NSEA).

La segunda categoría, Aceptación de la homosexualidad, alude al rechazo de los jóvenes frente al mandato de la heterosexualidad como condición de la masculinidad: "Yo creo que está mucho más asumido que un hombre por ser homosexual no deja de ser hombre" (J-NSEA); "...yo creo que para asumirse como homosexual, hay que ser bien hombre, tener los cojones bien puestos" (J-NSEM); “...si eres homosexual o no, no quiere decir que no seas hombre" (J-NSEB); "...un hombre debe ofrecer protección, no es tanto si estás con un hombre o con una mujer, sino cómo te vas a desenvolver en la sociedad y cumplir estos roles de protección, seguridad, tener un rol parental que igual puede tener un homosexual, etcétera, no creo que sea una condicionante ser homosexual" (J-NSEM).

La tercera categoría, Divergencias frente al mandato de la heterosexualidad, aparece solo en los grupos adultos y se refiere a la coexistencia de diferentes niveles de aceptación frente a la heterosexualidad como condición de la masculinidad: “...yo pienso que no, el homosexual, cómo va a ser hombre si es homosexual, no puede tener ese nombre de hombre porque le gustan los hombres" (A-NSEB); “...o no lo acepto, siendo de ningún credo religioso, llámese cristiano, yo me voy por el lado natural y el lado natural, como dijo un español, suena grotesco a lo mejor, pero dice 'pos hombre, el culo es pa' cagar"” (A-NSEM); “...y el sistema está haciendo eso con el ser humano, distorsionando lo que corresponde al hombre desarrollarse como hombre y a la mujer como mujer, en todo sentido" (A-NSEM); "Yo creo que bueno, el hecho de asumir de tener valor de decir las cosas, yo creo que también es como ser bien hombre para afrontarlo, quizás no sexualmente puede ser catalogado, pero sí por la valentía de asumir, por eso se podría decirse que sí" (A-NSEB); “...yo creo que el homosexual es hombre, ahora, qué tan varonil pueda ser en el sentido del concepto formal o estereotipado de la masculinidad, eso es lo que se puede relativizar, pero evidentemente un homosexual es un hombre, para mí" (A-NSEA).

La cuarta categoría, Estereotipos en el comportamiento sexual aparece fundamentalmente en los jóvenes de NSEA y NSEM y se refiere a la existencia de comportamientos sexuales diferentes en hombres y mujeres: “... se ve como un estereotipo generalizado de que el hombre queda como bien si engaña a la mujer, y la mujer queda como mal vista al engañar, la mayoría de las personas apoyan más la infidelidad de un hombre que de una mujer” (J-NSEA); “...el hombre en sí es más sexual que la mujer, creo yo, es otra forma de la sexualidad que tiene el hombre que la mujer, la mujer es más emotiva, el hombre dentro de todo es más carnal” (J-NSEM); “... a fin de cuentas el hombre es un animal, que es racional, lo que quieran, pero no deja de ser un animal entonces, como hombre, macho, está cableado para reproducirse la mayor cantidad de veces que pueda, entonces tiene menor autocontrol y si le sumamos que el hombre no carga con una cría por 9 meses, el hombre tiene cero autocontrol" (J-NSEA).

La quinta categoría, Comportamientos sexuales en transición, se refiere a la percepción en el grupo adulto de NSEA y a la experiencia de los jóvenes de NSEM de mayor naturalización y apertura en el ámbito sexual: "...las últimas generaciones, la cantidad de chicos de su generación que se asumen homosexuales es mucho mayor a los que se 
asumían en generaciones anteriores, dentro de una misma cohorte de una generación de amigos, cuatro, cinco o seis chicos hombres que pueden llegar a ser el $50 \%$ incluso, comienzan a manifestarse homosexuales $\mathrm{y}$ lo que veo en los estudiantes universitarios, y ellos lo dicen, que hay una fase no sexual, de no definición sexual, de pansexual, donde se explora y todo está permitido, y eso me sorprende" (A-NSEA); "...nosotros en eso, yo creo que hemos cambiado muchísimo, eso hace 40 años atrás se podría haber dado, 50 años atrás; pero ahora es cosa de prender la televisión a cualquier hora del día y hay una exaltación del cuerpo, en este punto ya se aceptan las relaciones prematrimoniales y todo como parte del paisaje" (A-NSEA); " el hombre ha ido dejando de ser peludo, hediondo y feo, entonces ha ido tomando una parte femenina, un ámbito femenino de autocuidado, que tiene relación directamente con su cuerpo y su sexualidad" (A-NSEA); “... pero igual hoy se admite caleta que el hombre diga, no hoy no quiero, hoy me duele la cabeza, de hecho se da caleta, pero cuando uno está en altos niveles de estrés es como que se permite decir que no, se permite que se dé su pausa" (J-NSEM); “...creo que antes, como que el hombre siempre cortejaba, iba a buscar a la mujer y todo el cuento y ahora por ejemplo no, vas a una disco y es la mujer la que se te lanza, la mujer es la que anda ahora como en el mismo tono tuyo, con unos tragos arriba y como a la par que el hombre" (J-NSEM).

\section{Discusión}

Es relevante destacar el interés de los participantes, particularmente en el grupo adulto de nivel socioeconómico alto, por compartir sus vivencias con otros varones, lo que podría reflejar la necesidad de reflexionar acerca de sí mismos y la falta de espacios para ello. Tal como se esperaba, se encontraron coincidencias y diferencias en los significados asociados a la masculinidad en función de la edad y nivel socioeconómico. Las coinci- dencias encontradas en el núcleo de Identidad dan cuenta de que los varones, independientemente de su edad o condición, vivencian una situación de cambio acelerada en sus roles que los afecta en mayor o menor medida, más allá de su identificación o no con esos cambios.

En cuanto al núcleo Trabajo, no obstante la amplia coincidencia en todos los grupos en relación con ser proveedor, se aprecian ciertos matices: para los adultos es un mandato interno, un deber ser de la masculinidad, mientras que para los jóvenes parece responder a una exigencia social externa, lo que da cuenta de una masculinidad en transición.

Las diferencias, por otra parte, encontradas en el núcleo Sexualidad entre jóvenes y adultos coinciden con lo señalado por Iñiguez (2001) quien plantea que la generación actual ha vivido, a diferencia de sus padres, en un contexto sociohistórico de mayor liberalidad y apertura, lo que ha permitido el desarrollo de actitudes y comportamientos más inclusivos.

Con respecto al núcleo Identidad, todos los grupos comparten que el concepto de "hombre" actual se encuentra en transición, que existen múltiples formas de ser hombre, lo cual es vivenciado por la mayoría de los jóvenes como una posibilidad de trascender los estereotipos, permitiéndoles mayores grados de libertad. Para algunos jóvenes, sin embargo, esta diversidad de modelos constituye una fuente de incertidumbre y ansiedad, lo cual daría cuenta de diferencias individuales más que diferencias socioeconómicas, dado que se expresaron al interior de los distintos grupos socioeconómicos (Olavarría, 2017). Lo anterior no se expresa en el grupo adulto, para quienes la existencia de diferentes formas de ser hombre no constituye una amenaza a una identidad ya consolidada.

La dificultad de ser hombre aparece fundamentalmente en adultos de NSEA, quienes lo describen como un proceso de tensión y exigencia permanente para cumplir los mandatos de la masculinidad hegemónica, siempre esquiva, por lo que la conciencia de esta dificultad, de acuerdo con Bourdieu, no 
hace más que fomentar la fragilidad del hombre: "Todo contribuye así a hacer del ideal imposible de la virilidad el principio de una inmensa vulnerabilidad" (Bourdieu, 2012, p. 69). En el discurso de los varones de NSEB no hay referencia a esta dificultad, lo que podría indicar que este grupo, consciente de la brecha entre su situación social y laboral y los mandatos de éxito económico y social, ni siquiera se plantean este desafío. Frente a ello, Kaufman (1989, citado por Botello, 2005) sugiere que uno de los riesgos de la imposibilidad de cumplir los mandatos es la utilización de los mecanismos compensatorios para sentirse valorados, como el ejercicio del poder o de la violencia hacia la mujer y/o los hijos/as.

Frente a la dificultad de consensuar elementos identitarios, llama la atención que aún permanece como definición esencial de la masculinidad, tanto en grupos de jóvenes como de adultos, la representación del hombre como un sujeto activo en contraposición a las mujeres, orientado al mundo externo, con capacidad de liderazgo asociado principalmente a la resolución de problemas, logro de objetivos y autonomía, derivada de las concepciones freudianas de comienzo de siglo XX (Freud, 1932, citado por Napolitano y San Juan, 2005). La integridad, por otra parte, como característica identitaria es señalada principalmente por los adultos, quienes destacan valores interaccionales como el compromiso y el respeto como fundamento de las relaciones. Para los jóvenes estos valores parecen ser menos significativos, lo cual se asocia posiblemente al haber crecido en una sociedad competitiva que prioriza el éxito individual por sobre el bienestar colectivo (Sandoval, 2011).

Por otra parte, en el plano de la expresión emocional, si bien los jóvenes reconocen mayor apertura social a la expresión de sus emociones, particularmente aquellas connotadas como debilidad, al mismo tiempo dan cuenta de la persistencia del mandato genérico que señala que deben mantenerlas en privado; la expresión más representativa de esta dimensión es que los hombres no lloran en público.
Al respecto, Bard-Widgor (2016) señala que este control sobre las emociones y afectos les permitiría a los varones protegerse y ejercer dominio sobre las mujeres, particularmente en una sociedad en la que se menosprecian los atributos femeninos y se enaltecen los masculinos como la racionalidad.

Respecto del núcleo Trabajo es interesante señalar que la condición de proveedor principal sigue siendo un mandato central de la masculinidad, considerando que es la categoría que más peso tiene dentro del análisis y que cruza todos los niveles etarios y socioeconómicos. Aun cuando los varones reconocen y valoran el ingreso mayoritario de las mujeres al mundo laboral y el aporte de ellas al ingreso familiar, persiste como un límite inmodificable la posibilidad de ser mantenido; los adultos lo perciben como un menoscabo a su dignidad y hombría, mientras que para los jóvenes la adhesión a este mandato está condicionada por la fuerte sanción social que implica la transgresión (Padilla y Echevarría, 2017).

El trabajo como facilitador de la independencia está condicionado por el nivel socioeconómico. Los jóvenes del NSEA y NSEM perciben que la disponibilidad de recursos les permite hacerse cargo de sí mismos, incluso en situaciones de dependencia relativa al residir en casa de sus padres. Aquí se aprecia una diferencia significativa con los jóvenes de NSEB, para quienes la disponibilidad de recursos económicos supone el logro de su independencia, y también hacerse cargo de sus padres, respondiendo a un deber de reciprocidad (Palomar y Victorio, 2016).

Es preocupante constatar que, si bien tanto jóvenes como adultos están conscientes de la jerarquía masculina y la discriminación laboral en relación con la mujer, no se aprecia frente a ello un discurso crítico, asumiéndolo como un hecho natural que no los moviliza. Esta actitud daría cuenta de una masculinidad cómplice, entendiendo por ella a aquellos varones que, si bien no forman parte de la minoría hegemónica, disfrutan de las ventajas del sistema patriarcal y no están dispuestos a ceder privi- 
legios (Bard-Widgor, 2016; Connel y Messerschmidt 2005).

Por último, en el ámbito del trabajo los resultados coinciden con lo señalado por De Keijzer (2001) y Conell (2003) con respecto al impacto que el trabajo físico tendría en el cuidado de la salud, la relación de pareja y el ejercicio de la sexualidad. Para los varones de NSEB, las exigencias físicas asociadas a su condición de trabajadores no calificados son tan demandantes que interfieren en la satisfacción de cualquier otra necesidad, dando cuenta de las enormes diferencias en las condiciones laborales en Chile.

Con respecto al núcleo Sexualidad, llama la atención la coexistencia de visiones en los jóvenes, la adhesión a estereotipos de género, como la imposibilidad de controlar el impulso sexual, justificado en el determinismo biológico de la conducta sexual. Esta creencia, si bien no mayoritaria, constituye un riesgo de comportamiento abusivo, lo que implica un desafío para los programas de prevención de la violencia sexual. Por otra parte, particularmente dentro de los jóvenes de nivel medio y alto, la percepción de exigencia en el plano sexual y el temor de no responder adecuadamente darían cuenta de un cambio en los roles de género y una percepción de la mujer más empoderada en el ámbito sexual (Hernández y González, 2016). Un avance importante, sin embargo, en los jóvenes respecto de los adultos, es la aceptación transversal de la homosexualidad como una expresión legítima de la sexualidad masculina, sin que ello signifique que un hombre pierda su condición de tal, a diferencia de los adultos en quienes coexiste aceptación y rechazo en todos los niveles socioeconómicos, lo que sugiere que la aceptación en este grupo etario estaría más relacionada con experiencias individuales que con variables socioculturales. Lo anterior podría reflejar, como lo señala Barrientos (2016), una creciente apertura de la sociedad chilena a las minorías sexuales. Finalmente, esta investigación da cuenta de la continuidad y ruptura respecto de los mandatos de la masculinidad hegemónica, así como de la coexistencia de demandas diversas y contrapuestas hacia los varones que se representan en el discurso de los participantes en la expresión "es difícil ser hombre". Como limitaciones es necesario mencionar el uso de los grupos de discusión como técnica de recolección de datos, el cual si bien facilita el acceso a la subjetividad de los participantes, tiende a favorecer el consenso dentro del grupo, minimizando las divergencias (Smithson, 2000).

Constituye un desafio para futuras investigaciones acceder a estos significados en hombres provenientes de distintas regiones del país, considerando la variabilidad cultural asociada a los diversos sectores geográficos y productivos, de pueblos originarios y población migrante, dado que los resultados solo representan a la población participante en este estudio. Los estudios sugeridos contribuirán a profundizar en la comprensión del fenómeno y orientar el diseño de políticas públicas.

\section{Referencias bibliográficas}

Addis, M. E. y Cohane, G. H. (2005). Social Scientific Paradigms of Masculinity and their Implications for Research and Practice in Men's Mental Health. Journal of Clinical Psychology, 61(6), 633-647. Recuperado de https://www2. clarku.edu/faculty/addis/menscoping/files/ addis_cohane_2005.pdf

Aguayo, F., Correa, P. y Cristi, P. (2011). Encuestas Images Chile. Resultados de la encuesta internacional de masculinidades y equidad de género. Santiago, Chile: CulturaSalud/EME. Recuperado de http://bit.ly/2ufMEUH

Aguayo, F. y Nascimento, M. (2016). Presentación: Dos décadas de Estudios de Hombres y Masculinidades en América Latina. Avances y Desafíos. Sexualidad, Salud y Sociedad. Revista Latinoamericana, 22, 207-220. http:// doi.org /10.159 0/1984-6487.sess.2016.22.09

Aravena, A. y Baeza, M. (2010). Jóvenes chilenos y construcción socioimaginario del ser otra mujer. Última Década, 32, 159-171. http://doi. org/10.4067/S0718 22362010000100009.

Bard-Widgor, G. (2016). Aferrarse o soltar privile- 
gios de género: sobre masculinidades hege-mónicas y disidentes. Península, 11(2), 101-122. https://doi.org/10.1016/j.pnsla.2016.08.003

Barrientos, J. (2016). Homofobia en Chile: una revisión del estado del arte. LIMINALES. Escritos sobre Psicología y Sociedad, 1(9), 129-151. Recuperado de http://revistafacso.ucentral.cl/index.php/liminales/article/ view/381.

Barker, G. y Greene, M. (2011). ¿Qué tienen que ver los hombres con esto?: Reflexiones sobre la inclusión de los hombres y las masculinidades en las políticas públicas para promover la equidad de género. En Aguayo F. y Sadler M. (Eds.), Masculinidades y Politicas Públicas. Involucrando hombres en la equidad de género (pp. 23-48). Santiago de Chile: FACSO, CulturaSalud/EME.

Botello, L. (2005). Identidad, masculinidad y violencia de género. Un acercamiento a los varones jóvenes mexicanos. (Memoria para optar al grado de Doctor). Facultad de Psicología, Universidad Complutense de Madrid, España. Recuperado de https://biblioteca.ucm. es/tesis/psi/ucm-t28455.pdf

Bourdieu, P. (2012). La dominación masculina. Barcelona: Anagrama.

Butler, J. (2006). Gender Trouble: Feminism and the Subversion of Identity. New York: Routledge

Connell, R. W. (2003). Masculinidades, Programa Universitario de Estudios de Género. Universidad Nacional Autónoma de México: México, D.F.

Connell, R. W. (2005). Masculinities. 2nd ed. Berkeley, CA: University of California Press.

Connell, R. W. (2011). Gender and Social Justice: Southern Perspectives, South African. Review of Sociology, 42 (3), 103-115. https://doi.org/1 $0.1080 / 21528586.2011 .621242$

Connell, R. y Messerschmidt, J. (2005). Hegemonic Masculinity: Rethinking the Concept. Gender y Society, 9(6), 829-859. https://doi. org/10.1177/0891243205278639

De Keijzer, B. (2001). Hasta donde el cuerpo aguante. En Cáceres C. et al. (Eds.), La salud como derecho ciudadano: perspectivas $y$ propuestas desde América Latina (pp. 1-26), Lima, Perú: Universidad Peruana Cayetano Heredia.

De Keijzer, B. (2010). Masculinidades, violencia, resistencia y cambio (Doctoral dissertation, Universidad Veracruzana, Veracruz, México). Recuperado de https://es.scribd. com/doc/207029466/Tesis-Benno-de-Keijzer-Masculinidades-Violencia-Resistencia-y-Cambio-2010.

Fuller, N. (2005). Identidades en tránsito: femineidad y masculinidad en el Perú actual. En T. Valdés y X. Valdés (Eds.), Familia y vida privada: ¿transformaciones, tensiones, resistencias y nuevos sentidos?, 107-125. Santiago de Chile: FACSO.

Goldscheider, F., Bernhardt, E. y Lappegård, T. (2014). Studies of Men's Involvement in the Family - Part I: Introduction. Journal of Family Issues. 35(7), 879-890. https://doi. org/10.1177/0192513X14522237.

Hernández, R., Fernández, C. y Baptista, P. (2010). Metodología de la Investigación. México: Mc. Graw Hill.

Hernández, A. y Fernández, J. (2016). Los roles $\mathrm{y}$ estereotipos de género en los comportamientos sexuales de jóvenes de Coahuila, México: aproximación desde la Teoría Fundamentada. Ciencia Ergo Sum, 23(2), 1-11. Recuperado de https://www.redalyc.org/jatsRepo/104/10446094003/html/index.html

Iñiguez, L. (2001). Identidad: De lo personal a lo social. Un recorrido conceptual. En E. Crespo y C. Soldevilla (Eds.), La constitución social de la subjetividad. Madrid: Catarata.

Kaufman, M. (1997). Las experiencias contradictorias del poder entre los hombres, En: Valdés, T. y Olavarría, J. (Eds.) Masculinidad/es: poder y crisis. Santiago de Chile: Isis Internacional-Flacso.

Kimmel, M. (1997). Manhood in America: A cultural history. New York: The Free Press.

Kimmel, M. S. y Messner, M. A (Eds.). (2004). Mens lives (6th ed.). Boston: Allyn y Bacon.

Kilmartin, C. T. (2007). The Masculine Self (3rd edition). New York: Sloan Publishing.

Matamala, M. y Rodríguez, M. (2010). Estudio 
exploratorio sobre la identidad de género de hombres adolescentes pertenecientes al sector Barrio Norte de Concepción. Última Década, 18(33), 61-84. http://doi.org/10.4067/S071822362010000200005.

Napolitano, E. y San Juan, K. (2005). La construcción de la subjetividad femenina en Freud: un estudio de sexualidad y cultura. (Memoria para optar al título de Psicólogas). Facultad de Ciencias Sociales Universidad de Chile, Santiago de Chile. Recuperado de http://www. tesis.uchile.cl/tesis/uchile/2005/napolitano_e/ sources/napolitano_e.pdf

O’Neil, J. M. (2008). Summarizing 25 Years of Research on Men's Gender Role Conflict Using the Gender Role Conflict Scale: New Research Paradigms and Clinical Implications, The Counseling Psychologist, 36, 356-445. https://doi.org/10.1177/0011000008317057

Olavarría, J. (2003). Los estudios sobre masculinidades en América Latina. Un punto de vista. Anuario Social y Político de América Latina y el Caribe, 6, 91-98. Recuperado de http://bit. ly/2ufO4yv

Olavarría, J. (2017). Sobre Hombres y masculinidades "ponerse los pantalones". Santiago, Chile. Universidad Academia de Humanismo Cristiano. Recuperado de http://www. creaequidad.cl/wp-content/uploads/2017/12/

Sobre-hombres-y-masculinidades.-Ponerse-los-pantalones.pdf.

Organización Mundial de la Salud (OMS). (2006). Defining sexual health Report of a technical consultation on sexual health 28-31 January 2002. Ginebra: OMS

Padilla, M. y Echevarría, N. (2017). Narrativas sobre la organización familiar durante el desempleo masculino. Interdisciplinaria, Revista de Psicología y Ciencias Afines, 34 (2), 275-294. https://www.redalyc.org/articulo. oa? id=18054403003

Palermo, H. (2016). "Machos y brujas en la Patagonia". Trabajo, masculinidad y espacio de la reproducción. Antípoda. Revista de Antropología y Arqueología, (25), 99-119: http://doi. org/10.7440/antipoda25.2016.05.

Palomar, J. y Victorio, A. (2016). Expectativas laborales en la adolescencia: correlatos psicosociales. Interdisciplinaria, Revista de Psicología y Ciencias Afines, 33(1), 95-110. Recuperado de https://www.redalyc.org/articulo. oa? id=180/18049204006

Peretz, T. (2016). Why Study Men and Masculinities? A Theorized Research Review, Graduate Journal of Social Science, 12(3), 30-43. Recuperado de http://gjss.org/sites/default/ files/issues/chapters/papers/GJSS\%20Vol\%20 12-3\%20Peretz.pdf

Petersen, A. (2003). Research on Men and Masculinities. Some Implications of Recent Theory for Future Work. Men and Masculinities, 6(1), 54-69. https://doi. org/10.1177/1097184X02250843

Romero, M. (2017). Significado del trabajo desde la psicología del trabajo. Una revisión histórica, psicológica y social. Psicología desde el Caribe. Universidad del Norte, 34 (2), 120-138. Recuperado de https://dialnet. unirioja.es/servlet/articulo? codigo $=6552721$

Sandoval, M. (2011). La confianza de los jóvenes chilenos y su relación con la cohesión social. Ultima Década, 19(34), 139-165. https://doi. org/10.4067/S0718-22362011000100008

Smithson, J. (2000). Using and analysing focus groups limitations and possibilities. International Journal of Social Research Methodology, 3(2), 103-119. http://doi. org/10.1080/136455700405172

Taylor, S. y Bogdan, R. (2004). Introducción a los Métodos Cualitativos de Investigación. España: Paidós.

Vera, N. y Valenzuela, M. (2012). El concepto de identidad como recurso para el estudio de transiciones. Psicologia \& Sociedade, 24(2), 272-282. Recuperado de http://www.scielo.br/ pdf/psoc/v24n2/03.pdf

Recibido: 20 de diciembre de 2018 Aceptado: 24 de agosto de 2020 
